\title{
AXMEDIS architectural solution for interoperable content and DRM on multichannel distribution
}

\author{
Pierfrancesco Bellini, Sauro Chellini, Tommaso Martini, Paolo Nesi, \\ Davide Rogai, Andrea Vallotti \\ Distributed System and Internet Technology Lab, Department of Systems \\ and Informatics, University of Florence \\ nesi@dsi.unifi.it \\ WWW home page: www.axmedis.org
}

\begin{abstract}
AXMEDIS project (Automating Production of Cross Media Content for Multi-channel Distribution) is partially funded by the European Commission to create an innovative technology framework for the automatic production, protection and distribution of digital cross-media contents over a range of different media channels including PC (on the internet), PDA, kiosk, mobile phones and i-TV (interactive-TV). The AXMEDIS project has proposed a set of integrated solutions and technologies that covers data model and DRM. This paper presents a brief introduction to the AXMEDIS IST FP6 EC project, while discussing the new functionalities enabled by the AXMEDIS architecture and solution in terms of interoperable content and DRM among different distribution channels. For further details on the AXMEDIS project, see the project website at www.axmedis.org.
\end{abstract}

\section{Introduction}

In the evolving scenario of the digital content market, final users are asking content distributors for more functionalities to be exploited on the acquired content. At present, the simple solutions already available on the market such as i-Tune or Microsoft Media based solutions are mainly related to business models and DRM (Digital Rights Management) mechanisms, allowing to exploit a number of limited rights on the acquired digital content. For instance, they could limit the content usage in the platform the content has been bought with or they could have a limited flexibility in porting the content on CDs (limited number of burnings, etc.), some limitations when it comes to copying the content (e.g., for a limited number of times) or other devices (e.g., from one i-Pod to another), etc. This is the beginning of a new era, when users are becoming more and more interested in acquiring digital content which can be really exploited on several different devices and tools, in comparison with what

Please use the following format when citing this chapter:

Bellini, Pierfrancesco, Chellini, Sauro, Martini, Tommaso, Nesi, Paolo, Rogal, Davide, Vallotti, Andrea, 2006, in IFIP International Federation for Information Processing, Volume 204, Artificial Intelligence Applications and Innovations, eds. Maglogiannis, I., Karpouzis, K., Bramer, M., (Boston: Springer), pp. 697-704 
the users can do with the traditional physical media. A good analysis of the Traditional Rights Usage (TRU) has been carried out by DMP (Digital Media Project), http://www.dmpforg/s see [1] for a comparison of DMP and AXMEDIS. In fact, consumers are setting out to acquire digital content that can be freely moved from one device to another at their homes, be passed into their children's hands when needed, be transferred on their mobile smartphones or into their cars, be collected in a house Media Center, etc.

In order to satisfy these needs, several challenges have to to be solved such as flexible Digital Rights Management, dynamic content adaptation, content modeling, content production on demand, content licensing, content interoperability, DRM interoperability, license processing, etc.

With this aim, AXMEDIS IST FP6 integrated project of the European Commission (Automating Production of Cross Media Content for Multi-channel Distribution) has been started up in September 2004. One of the main objectives of AXMEDIS is to create and exploit innovative technological framework for automatic production and distribution of cross-media contents over a number of different distribution channels (e.g., networked PC, PDA, kiosk, mobile phone, i-TV, etc) with DRM (Digital Rights Management). The AXMEDIS consortium consists of leading European digital content producers, integrators, aggregators, and distributors, together with information technology companies and research groups (http://www, axmedis.org. [2]).

\section{AXMEDIS Main Features}

In AXMEDIS a set of tools for content production, processing, protection and management has been produced. The main functionalities of the AXMEDIS tools include:

- Automated content gathering/crawling from legacy content management systems: ODBC, XML, ORCALE, MSSQL, MYSQL, etc., [2].

- Content management database supporting the storage and access to AXMEDIS content (MPEG-21, any digital resource, etc., [2]) via a large set of metadata for each object called AXInfo, plus Dublin core, etc. Any other metadata format can be managed by the AXMEDIS metadata editors and tools. Different descriptors and metadata can be added into the AXMEDIS flexible model.

- Automated content processing, processing metadata and digital resources, extracting fingerprint, watermark, content synchronization, adaptation (change in resolution and format, Transcoding, etc.), coding/decoding, estimation of descriptors, license production and processing, content protection, publication, packaging, formatting, loading/saving, etc. These features are provided by means of AXMEDIS Editors and via the so-called AXMEDIS Content Processing GRID which is based on and a specific AXMEDIS extension of ECMA Script language [3]. It is a scalable solution for automating and accelerating all the phases of content processing, also integrated with Open Flow workflow.

- Editing AXMEDIS objects with a set of authoring tools. It is based on the AXMEDIS Object Model, called AXOM and extending MPEG-21 [5], and all the modules and tools to manipulate and create AXMEDIS objects and related information and digital resources such as: (i) a resource hierarchy viewer and editor, (ii) a visual and behavioral viewer and editor to show/manipulate visual and time aspects of digital resources, (iii) a DRM 
viewer and editor, (iv) a protection information tools, (v) a set of plug-ins to use algorithms for content processing, (vi) a set of plug-ins to allow the integration of AXMEDIS Editor within other editing and viewing applications, (vii) a set of internal viewers and players for digital resources such as document, images, video, audio resources, etc., for more than 300 different file formats.

- Viewing and playing AXMEDIS objects with specific tools that support large set of possible digital resources, together with different business and transaction models. At present mainly on $\mathrm{PC}$, while the same tools will be available on MAC, Linux, PDA, and maybe on mobiles. The current players are also usable as plug-in of Internet Explorer (ActiveX) and Mozilla.

- Automating distribution solutions at B2B level supporting both P2P and Client/Server models, automating publication and download of digital content from and to a P2P network for B2B distribution.

- Automating distribution solutions at B2C levels supporting both P2P and Client/Server models. Any third party distribution tool and solution can be used to distribute AXMEDIS content on their distribution channels. The usage of the AXMEDIS tools to realize different distribution channels and business models has already been tested: satellite data broadcast, Internet, cellular networks, wireless from kiosks, etc., to reach devices such as: $\mathrm{i}-\mathrm{TV}$ PC, PC, PDA, mobiles, etc.

- Authentication, registration and certification of users and tools.

- Supervision and control of the exploitation of licensed rights by means of the AXMEDIS Certifier and Supervisor (AXCS) and the AXMEDIS Protection Manager Support (PMS).

All the above functionalities are available by means of a set of tools, libraries, solutions, guidelines, etc., that belong to the AXMEDIS Framework, AXFW (the specification is accessible on www.axmedis.org ), [2]. The AXFW contains the necessary tools to set up distribution channels and make them interoperable. The AXFW includes: requirements, test cases, use cases, content for validations, general documentation of AXMEDIS tools and supports, source code, guidelines for source code production, state of the art analyses, market analyses, comparison with other technologies, guidelines on content production and distribution, tutorials on content protection, tutorial on AXMEDIS tools, etc. It is possible to get access to the AXMEDIS Framework by means of subscription/affiliation. Specific events are organised to present AXMEDIS technologies. Further information is available online at the project website, www.axmedis.org Furthermore, the AXMEDIS consortium will grant the sum of 1 Million Euro by means of a European competitive call to companies and research institutes interested in developing real solutions by exploiting AXMEDIS technologies.

\section{AXMEDIS Model Flexibility}

In order to guarantee interoperability of content and DRM, the most relevant aspect has to do with content model. In AXMEDIS several different content models can be adopted and the AXMEDIS tools may support all of them. As a general rule, the relevant elements of any protected digital content are digital resources, metadata, any kind of information needed to unprotect the objects and their related license. In AXMEDIS these elements may be managed in an independent way:

Metadata as AXMEDIS Information, AXInfo:

- Identification information, object unique ID, distributor ID, etc. 
- Classification information also for indexing: Dublin core, etc.

- Descriptors, MPEG-7, for indexing, etc.

- References to content owner, to distributor, etc.

- Potential available rights, PAR, formalised in MPEG-21 REL.

- Etc.

Digital Resources:

- Any digital information: images, doc, txt, video, game, application, file, audio, etc.

- Hierarchy of digital resources according to MPEG-21 Digital Item model.

Protection Information, Prot.Info (IPMP information):

- What should be done to get access to a given information/resource.

- Tools used, their parameters, etc.

- extended version of MPEG-21 IPMP.

License:

- Which rights are provided, who is the recipient, which conditions, etc.

- MPEG-21 REL compliant, with some specific profile.

Usually, metadata and digital resources are combined in different ways, according to the production model used. In some cases, some metadata and/or digital resources are protected, whereas other ones may be left accessible, meaning unprotected. These combinations allow to create a complex content with promotional information and resources as well. The combination of metadata and digital resources is typically called the "Content".

In many DRM models, the content is protected and the specific Protection Information (which is called 'IPMP information' in some models like for istance MPEG) is stored to allow any opening of the object; the simpler solution is the key to decrypt the encrypted object. In AXMEDIS, the Protection Information can be both a complex set of instructions with the related protection tools to unprotect the object and also each single digital resource, beginning with the stream and/or the file on the disk.

The License is a sort of digital contract between the one who is selling the content (providing access to some specific set of rights) and the other party who is going to use/exploit them. It codes in some way the business model, and includes the rights which can be exploited by the users on a specific content, and it is formalized in some formal and consistent language such as ODRL (OMA), XrML, MPEG-21 REL, as in the AXMEDIS. The language is based on a dictionary of terms for defining the semantics of rights and related constraints (such as MPEG-21 RDD). In AXMEDIS, each license is assigned to a user, or to a group of them, or to a device or to a domain/set of devices. Any kind of business model can be implemented: pay per view, monthly rate subscription, all you can eat, pay per renting, pay per print, pay per stream, download, burning the $\mathrm{CD}$, transcoding for migrating content on different devices or distributing them on different channels, etc. Supporting different constraints (number playing, temporal windows in which can be seen, expiration date, etc.) and additional features such as massing copies, building a collection, preview without paying, try and buy, etc. Before providing the Protection Information to open a given digital resource, the related Grant of the License has to be estimated to verify if the user has the right to get access to those contentrelated functionalities.

Each Grant of a given license may depend on the grants in other Licenses. For instance, a distributor may have received a License A for sublicensing (producing Licenses B) to its customers. On such grounds, each grant of License B is estimated if and only if the License A 
is accessible and can be positively processed, for instance on a License Server. This model produces a chain of Licenses that can be located in one or more servers.

In some solutions the Licenses and/or the Protection Information are cached on the device in some hidden location. This solution is a way to allow the exploitation of rights, when the device is not connected to the License Server, at the expenses of the security level.

The Protection Information and the License can be managed in different ways with respect to the metadata and digital resources. Mainly, three different models are possible:

Open Model: the Content is protected and the Protection Information is produced, while several different Licenses can be produced according to the business models and to the final users (see Fig.1). The Distributor has to produce a License for each final user or a group of them. Therefore, if the Distributor has $O$ objects and $U$ users, it may have $O * U$ Licenses and only O Protection Information, one for each object. This model is suitable for P2P distribution, since the Objects can be freely distributed and when the user is interested in opening/playing one of them, he/she has to acquire a License which is the only way to obtain access to the Prot.Info.

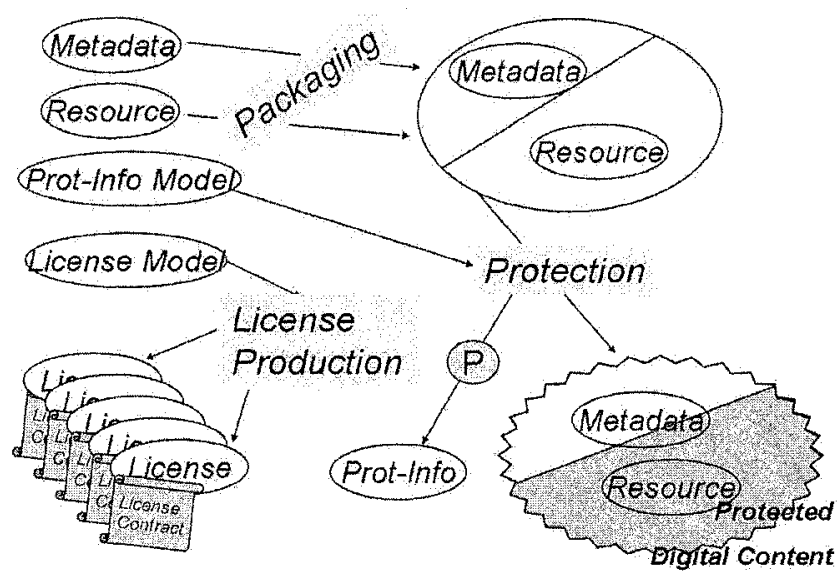

Fig. 1. Open Model (the copyrights of this figure belong to DSI AXMEDIS)

Governed Object: the Content is protected including the License inside. On such grounds, each object should be produced for each user. Therefore, if a Distributor has $\mathrm{N}$ objects and $\mathrm{U}$ users, it has to produce $\mathrm{N}^{*} \mathrm{U}$ Objects and Protection Information. This solution is very expensive to be realized, since the $\mathrm{N}^{*} \mathrm{U}$ Objects take a lot of space. In this case, for managing the same number of objects of the Open Model, a space larger $U$ times is needed. If the needed number of objects is not produced the License is not associated with the User and it is larger and less precise, while the Objects can be passed on to other Users of the same group, or as a limit case the user in the License included is anonymous. This decreases the security level of the solution. This model is not suitable for P2P distribution, since the objects contain personal information about who bought them.

Augmented License: the Content is protected and the Protection Information is produced, while several different Licenses can be produced according to the business models and to the 
final users .Each License contains the Protection Information. The Distributor produces a License for each final user or for a group of them. Therefore, if the Distributor has O objects and $U$ users, it may have $O^{*} U$ Licenses. This model is suitable for P2P distribution since the Objects can be freely distributed and when a user is interested in opening one of them, he has to acquire the License. This model presents more risks than the Open Model since the License contains the protection information and frequently the License has to be visible and accessible to the final user.

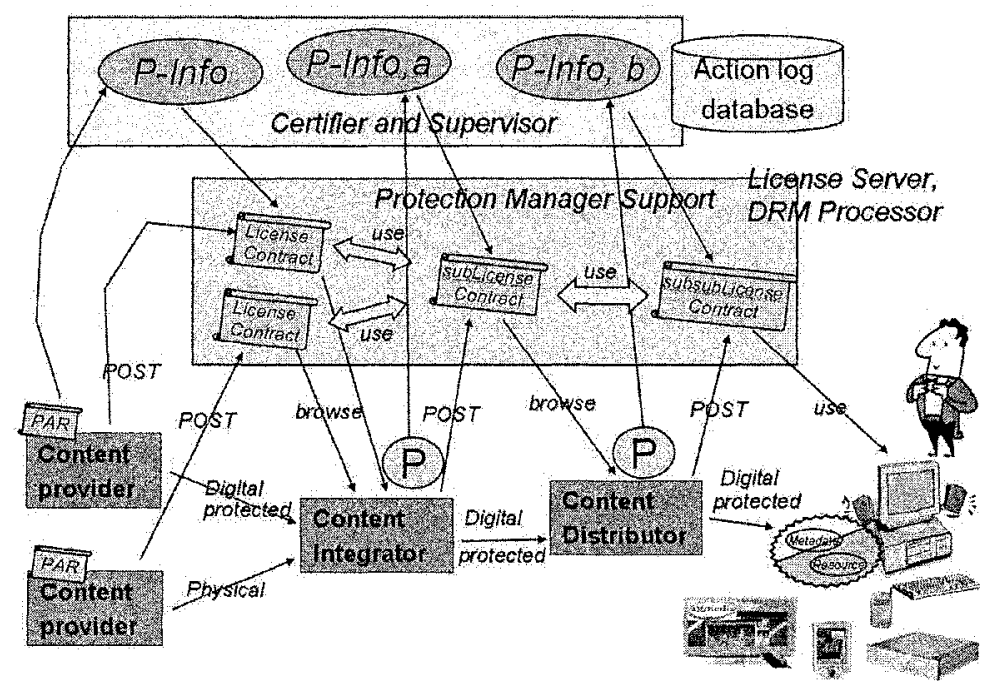

Fig. 2. Processing Licenses (the copyrights of this figure belong to DSI AXMEDIS)

In AXMEDIS, the distributors and in general the producers of protected objects are free to decide which model they prefer to adopt, according to their business and transaction models. The Open Model is most flexible and it allows to manage in an independent way the chain of licenses and the management of the Protection Information (see Fig.2). To this end, the AXMEDIS Protection Manager Support (PMS) and the AXMEDIS Certifier and Supervisor (AXCS) are provided.

The AXCS collects and maintains the information regarding the registered objects, users, devices, etc, and therefore it allows the management of black lists. It also stores the Protection Information of each protected object, resource and the list of actions performed on them, the so called Action Log database. Each Action describes an action performed on a given content/resource, by a given user, on the basis of a given license, etc. The whole set of Action Logs allows to produce the reporting to Distributors, to Content Providers, to Collecting Societies, etc., and also the production of statistics on the use of digital content per area, per device, per genre, per type, per sex, per device type, in a given period, etc.

The PMS is a License Server that maintains the database of Licenses and it is capable of processing chains of them for evaluating the Grants. 


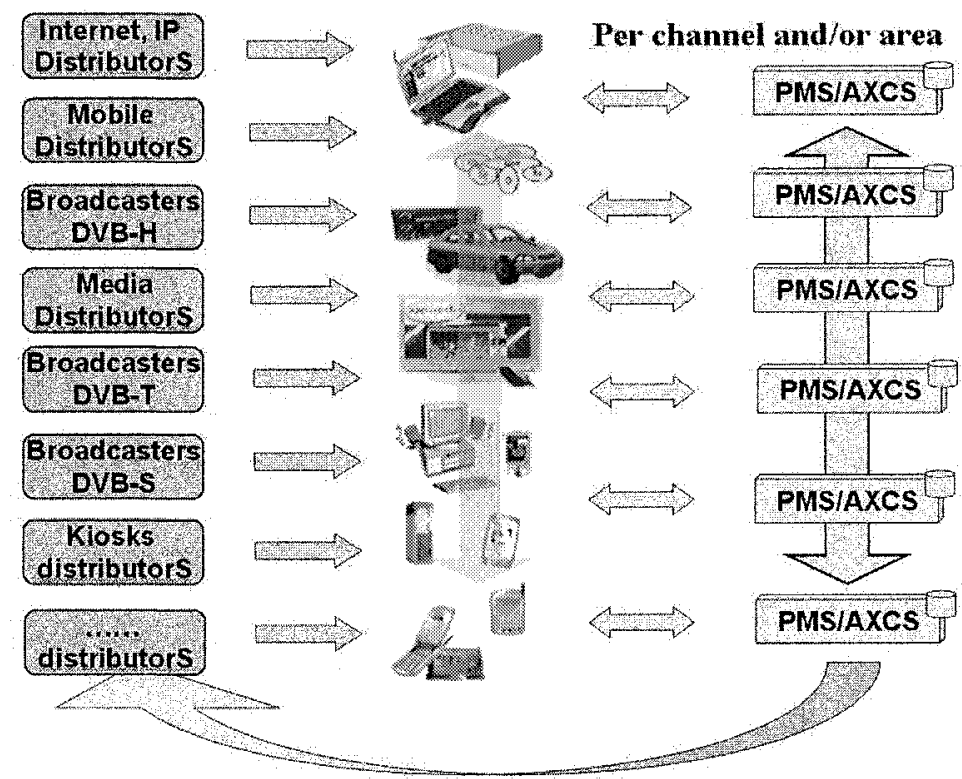

Fig. 3. AXMEDIS Multichannel (the copyrights of this figures is of DSI AXMEDIS)

The AXMEDIS architecture supports multichannel and interoperability by allowing the migration of content from different devices and among different channels. Such different channels may have their specific devices and business models and their specific DRM solutions. Each of them can be a customization of MPEG-21 REL or OMA ODRL. This means that the different PMS/AXCS managing the different geographic areas or channels have to communicate one another to exchange information about the: Actions Log, registered objects, Protection Information, Licenses, etc. In addition, the local AXCS has to provide access to the whole information stored in the Action Logs related to the usage of a given content or to a given content owner or distributor. The flow of this information is the most important problem concerning the interoperability of content and DRM. It has to put up with any possible failure of some PMSs or AXCSs.

\section{Conclusions}

AXMEDIS architecture and tools are a solution to automate, accelerate and restructure production and protection processes. This paper described a single aspect of the whole AXMEDIS architecture and framework design which is going to address many other problems and critical points. AXMEDIS framework can support data gathering from accessible Content Management Systems, transform legacy digital content in AXMEDIS objects, content authoring, and it can process them in the production, preserving security level along the whole value chain and therefore creating a perfect environment for content production, protection and distribution at both $\mathrm{B} 2 \mathrm{~B}$ and $\mathrm{B} 2 \mathrm{C}$ levels. AXMEDIS solution is mainly based on MPEG- 
21 model and it provides and stimulates the usage and the exploitation of the developed features for creating many AXMEDIS compliant tools and solutions, while making the core aspects and solution accessible in the form of AXMEDIS Framework. More technical information and/or how to make registration or submit affiliation to the AXMEDIS can be found on www.axmedis.org

\section{Acknowledgements}

The authors would like to thank all the AXMEDIS project partners (ANSC, AFI, EUTELSAT, Giunti ILABS, HP Italy, FHGIGD, DIPITA, CRS4, TISCALI, XIM, ACIT, FUPF, CPR, EXITECH, Univ of Leeds, etc.), the Expert-User-Group and all affiliated members for their contributions, supports and collaborations. The authors would also like to express their thanks to the EC IST FP6 for partially funding the AXMEDIS project.

\section{References}

1. AXMEDIS, "Comparing AXMEDIS, MPEG21 and DMP", accessible at www.chiarigllione.org and on WWW.AXMEDIS.org

2. AXMEDIS Use Cases, Official Deliverable of AXMEDIS project: www axmedis.org.

3. P. Bellini, I. Bruno, P. Nesi, "A. Distributed Environment for Automatic Multimedia Content Production based on GRID", Proc. of International Conference on Automated Production of Cross Media Content for Multi-channel Distribution, 30 November - 2 December 2005, Florence, Italy.

4. P. Bellini, P. Nesi, "An architecture of Automating Production of Cross Media Content for Multi-channel Distribution", Proc. of International Conference on Automated Production of Cross Media Content for Multi-channel Distribution, 30 November - 2 December 2005, Florence, Italy.

5. P. Bellini, P. Nesi, D. Rogai, A. Vallotti, "AXMEDIS Tool Core for MPEG-21 Authoring/Playing", Proc. of International Conference on Automated Production of Cross Media Content for Multi-channel Distribution, 30 November - 2 December 2005, Florence, Italy. 